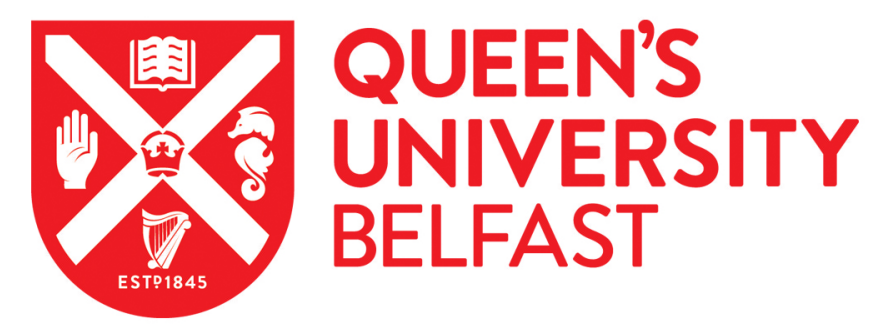

\title{
Detecting Positron-Atom Bound States through Resonant Annihilation
}

Dzuba, V. A., Flambaum, V. V., \& Gribakin, G. F. (2010). Detecting Positron-Atom Bound States through Resonant Annihilation. Physical Review Letters, 105(20), [203401].

https://doi.org/10.1103/PhysRevLett.105.203401

Published in:

Physical Review Letters

Document Version:

Publisher's PDF, also known as Version of record

Queen's University Belfast - Research Portal:

Link to publication record in Queen's University Belfast Research Portal

Publisher rights

(C) 2010 The American Physical Society

\section{General rights}

Copyright for the publications made accessible via the Queen's University Belfast Research Portal is retained by the author(s) and / or other copyright owners and it is a condition of accessing these publications that users recognise and abide by the legal requirements associated with these rights.

Take down policy

The Research Portal is Queen's institutional repository that provides access to Queen's research output. Every effort has been made to ensure that content in the Research Portal does not infringe any person's rights, or applicable UK laws. If you discover content in the Research Portal that you believe breaches copyright or violates any law, please contact openaccess@qub.ac.uk. 


\title{
Detecting Positron-Atom Bound States through Resonant Annihilation
}

\author{
V. A. Dzuba* and V. V. Flambaum ${ }^{\dagger}$ \\ School of Physics, University of New South Wales, Sydney 2052, Australia \\ G. F. Gribakin \\ Department of Applied Mathematics and Theoretical Physics, Queen's University, \\ Belfast BT7 1NN, Northern Ireland, United Kingdom
}

(Received 4 August 2010; revised manuscript received 23 September 2010; published 9 November 2010)

\begin{abstract}
A method is proposed for detecting positron-atom bound states by observing enhanced positron annihilation due to electronic Feshbach resonances at electron-volt energies. The method is applicable to a range of open-shell transition-metal atoms which are likely to bind the positron: $\mathrm{Fe}, \mathrm{Co}, \mathrm{Ni}, \mathrm{Tc}, \mathrm{Ru}$, $\mathrm{Rh}, \mathrm{Sn}, \mathrm{Sb}$, Ta, W, Os, Ir, and Pt. Estimates of their binding energies are provided.
\end{abstract}

PACS numbers: 36.10.-k, 34.80.Lx, 34.80.Uv, 78.70.Bj

Our analysis has identified about 25 open-shell atoms that are likely to form bound states with the positron. We show that for many of them binding can be detected through resonantly enhanced positron annihilation.

The existence of positron-atom bound states was predicted by many-body theory calculations [1] and proved variationally $[2,3]$ more than a decade ago. Since then positron binding energies have been calculated for many ground-state and excited atoms: $\mathrm{He} 2^{3} S, \mathrm{Li}, \mathrm{Be}, \mathrm{Be} 2^{3} P$, $\mathrm{Na}, \mathrm{Mg}, \mathrm{Ca}, \mathrm{Cu}, \mathrm{Zn}, \mathrm{Sr}, \mathrm{Ag}$, and $\mathrm{Cd}$ [4-9]. They range from $\sim 10 \mathrm{meV}$ to $\sim 0.5 \mathrm{eV}$.

In spite of this wealth of predictions, experimental verification of positron binding to neutral atoms is still lacking. To observe positron-atom bound states and measure their energies, one needs to produce sufficient numbers of atoms in the gas phase or in a beam, but more critically, to find an efficient way of populating these bound states. Thus, radiative recombination, $A+e^{+} \rightarrow e^{+} A+\hbar \omega$, is inefficient because of the small cross section, $\sigma \sim(\omega / c)^{3}$ (in atomic units in which $m_{e}=\hbar=e^{2}=1$ and the speed of light $c \approx 137$ ). One suggestion applicable to atoms with positive electron affinities, was to use a charge-transfer reaction for negative ions, $A^{-}+e^{+} \rightarrow e^{+} A+e^{-}$, and measure either its threshold energy or the electron spectrum [10,11]. The cross section of this process should be atomic-sized, but this scheme has not been realized experimentally yet.

In contrast, much is now known about positron binding to molecules. Binding energies for over 30 polyatomic species have been determined $[12,13]$ by measuring positron annihilation with a high-resolution, tunable, trapbased positron beam [14]. The key idea of this method is that for molecules that are capable of binding the positron, the dominant annihilation mechanism is through formation of positron-molecule vibrational Feshbach resonances [15-17]. The majority of the resonances observed are associated with individual vibrational modes of the molecule. The binding energy $\varepsilon_{b}$ can then be found from the downshift of the resonance energy $\varepsilon_{\nu}=\omega_{\nu}-\varepsilon_{b}$ with respect to the energy $\omega_{\nu}$ of the vibrational excitation $[18,19]$. These experiments proved the link between positron binding and enhanced annihilation rates [17].

For atoms existing theoretical predictions of positron binding are limited to species with one or two valence $s$ electrons, as these systems are easier to compute. It is expected that many other atoms with open multielectron valence shells, can bind the positron $[1,10]$. Physically, positron binding is facilitated by a sizeable dipole polarizability $\alpha_{d}$ and moderate ionization potential $I$. While there is no rigorous criterion for binding, examination of the atoms that bind, suggests the following conditions: $\alpha_{d} \gtrsim 40$ a.u. and $I<10 \mathrm{eV}$.

Large values of $\alpha_{d}$ ensure that the positron experiences a strong attractive polarization potential $-\alpha_{d} / 2 r^{4}$ outside the atom. Small ionization potentials increase the effect of virtual positronium (Ps) formation: a process in which an atomic electron temporarily joins the positron. It gives a distinct contribution to the positron-atom attraction akin to covalent bonding $[1,20,21]$. The energy of the ground-state Ps is $E_{1 s}=-6.8 \mathrm{eV}$, and this effect is strongest for $I \sim 6.8 \mathrm{eV}$. For atoms with $I<\left|E_{1 s}\right|$, positron bound states increasingly have the character of a "Ps cluster" orbiting the positive ion [22]. In this case the criteria for binding change, atoms with compact cores being favored (e.g., $e^{+} \mathrm{Na}$ is bound while $e^{+} \mathrm{K}$ is not). Atoms with $I<6.8 \mathrm{eV}$ also differ in one other important aspect: the Ps-formation channel $\left(A+e^{+} \rightarrow A^{+}+\right.$Ps $)$is open at all positron energies for them.

Figure 1 shows the polarizabilities vs ionization potentials for atoms with $6.6<I<10 \mathrm{eV}$. For most of them $\alpha_{d}>40$ a.u., and according to the above criterion, they are likely to form bound states with the positron. Solid symbols identify atoms for which the binding energies have been calculated: $\mathrm{Be}, \varepsilon_{b}=87$ [7]; Zn, 103 [9]; Cd, 126 [23]; $\mathrm{Ag}, 123$ [5]; $\mathrm{Cu}, 170$ [4]; and $\mathrm{Mg}, 464 \mathrm{meV}$ [9]. The weakest binding in this group is by $\mathrm{Be}$ and $\mathrm{Zn}$ found on the bottom right in Fig. 1. For a nearby atom of 


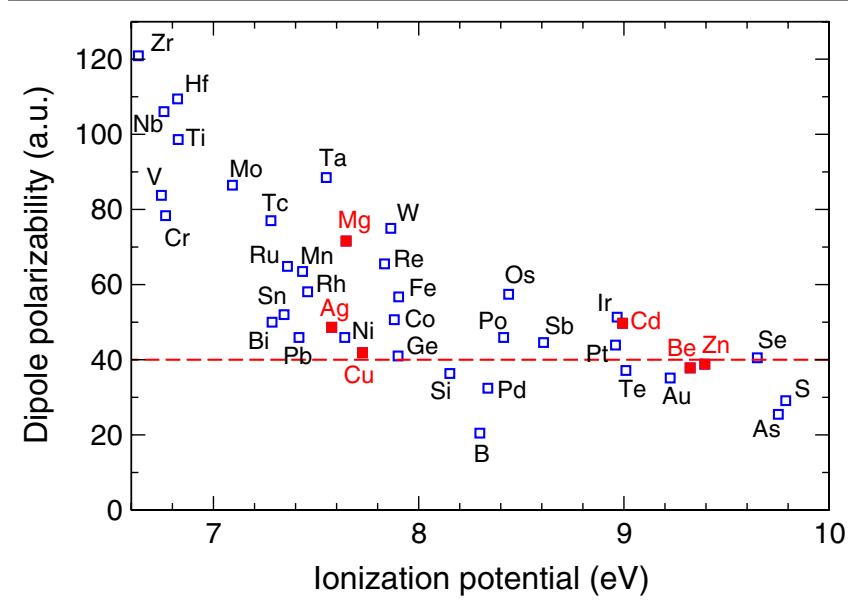

FIG. 1 (color online). Dipole polarizabilities $\alpha_{d}$ (from Ref. [25], except for Au [33]) vs ionization potentials for atoms in the range where positron binding can be expected. Solid squares show atoms for which positron binding is predicted by high-quality calculations. The horizontal line $\alpha_{d}=40$ a.u. is an approximate boundary between binding and nonbinding atoms.

gold positron binding occurs in the nonrelativistic approximation (which underestimates the ionization potential and overestimates $\alpha_{d}$ ). However, in a fully relativistic calculation this system is not bound [5].

Figure 1 shows that most good candidates for positron binding are transition-metal atoms with open $p$ or $d$ subshells. Many of these atoms possess low-lying excited states with energies $\sim 1 \mathrm{eV}$, due to a fine structure or Coulomb splitting of the ground-state configuration, or $n s-(n-1) d$ transitions. Their polarizabilities are similar to those of the ground states. Hence, they are also likely to bind the positron. Depending on the excitation and binding energies, this will be either a true bound state $\left(\varepsilon_{b}>\omega_{\nu}\right)$, or a resonance in the positron continuum $\left(\varepsilon_{b}<\omega_{\nu}\right)$.

To substantiate the claim that open-shell atoms can bind positrons, we have estimated their binding energies using many-body theory calculations. In this approach the positron wave function $\psi$ and energy $\varepsilon_{0}$ are found from the Dyson equation [1],

$$
\left(H_{0}+\Sigma\right) \psi=\varepsilon_{0} \psi
$$

where $H_{0}$ is the positron Hamiltonian in the field of the ground-state atom in the Hartree-Fock approximation, and $\Sigma$ is a nonlocal operator that describes the effect of correlations ("correlation potential"). In the lowest order of many-body perturbation theory $\Sigma$ is given by the 2nd-order contribution $\Sigma^{(2)}$ which accounts for the atomic polarization [24]. Higher-order contributions, such as that of virtual Ps formation, make the total correlation potential stronger. They are also more difficult to evaluate. To gauge their importance we solved Eq. (1) with $\Sigma=\zeta \Sigma^{(2)}$ and adjusted the coefficient $\zeta$ to reproduce the known binding energies for $\mathrm{Be}, \mathrm{Mg}, \mathrm{Zn}, \mathrm{Cd}, \mathrm{Cu}$, and $\mathrm{Ag}$, which yielded $\zeta=2.0$, $2.4,1.8,1.7,2.4$, and 1.9 , respectively. The advantage of this approximation is that it allows one to evaluate $\Sigma$ for open-shell atoms and excited states, by using orbital occupation factors in the calculation of $\Sigma^{(2)}$. Estimates of the binding energies for a number of open-shell atoms in the ground and excited states obtained using $\Sigma=\zeta \Sigma^{(2)}$ with $\zeta=2$, are listed in Table I. They range between $20 \mathrm{meV}$ and $0.5 \mathrm{eV}$. We have checked that even for a smaller value, e.g., $\zeta=1.7$, all the atoms in the table retain binding.

Table I also lists the energies of low-lying atomic excited states. The state of a positron bound to an excited atom, which lies above the atomic ground state, is a Feshbach resonance. Its total width $\Gamma$ is determined by the annihilation width $\Gamma^{a}$ and elastic width $\Gamma^{e}$. The latter gives the decay rate of this quasibound state into the $A+e^{+}$continuum, and also characterizes the probability of its formation in positron-atom collisions. Strong annihilation resonances require $\Gamma^{e} \gg \Gamma^{a}$ (see below).

An additional consideration used in selecting the atoms in Table I, was the energy of their Ps-formation threshold $E_{\mathrm{Ps}}=I-\left|E_{1 s}\right|$. If a positron resonance lies above $E_{\mathrm{Ps}}$, it can also decay into the $A^{+}+$Ps channel. This can significantly increase the total width, reducing the size of the annihilation resonance. More importantly, for incident positron energies $\varepsilon>E_{\mathrm{Ps}}$, Ps formation becomes the dominant annihilation channel, and the resonant annihilation signal is "drowned." Hence, we focus on resonances that lie below the Ps-formation threshold.

The last column in Table I indicates the type of electromagnetic transitions between the ground and excited states allowed by selection rules. All of the excited states have the same parity as the ground state. The majority of them have nonzero total angular momenta $J$. As a result, the most common allowed transition between the levels is E2. Of course, the excitation of an atom by the Coulomb field of the positron in the process of capture, is different from that by a photon. However, for the transitions of electric type, a simple estimate of the elastic width in terms of the atomic transition amplitude can be derived, which shows that $\Gamma^{e} \gg \Gamma^{a}$ (see below).

The resonant contribution to the positron-atom annihilation cross section is written using the Breit-Wigner formalism [27] as

$$
\sigma_{a}=\frac{\pi}{k^{2}} \sum_{\nu} \frac{2 J_{\nu}+1}{2 J+1} \frac{\Gamma_{\nu}^{a} \Gamma_{\nu}^{e}}{\left(\varepsilon-\varepsilon_{\nu}\right)^{2}+\Gamma_{\nu}^{2} / 4}
$$

where $k=\sqrt{2 \varepsilon}$ is the positron momentum, $J$ is the total angular momentum of the target ground state, and $J_{\nu}$ is that of the resonance $\nu$. To estimate the observable effect, we average the normalized dimensionless annihilation rate $Z_{\text {eff }}=\sigma_{a} k /\left(\pi r_{0}^{2} c\right)\left(r_{0}\right.$ being the classical electron radius) over the energy distribution in the positron beam, and obtain

$$
Z_{\mathrm{eff}}(\epsilon)=\frac{2 \pi^{2} \rho_{e p}}{2 J+1} \sum_{\nu} \frac{\left(2 J_{\nu}+1\right) \Gamma_{\nu}^{e}}{k_{\nu} \Gamma_{\nu}} \Delta\left(\epsilon-\varepsilon_{\nu}\right)
$$


TABLE I. Atoms with low-lying excited states in which positron binding and annihilation resonances are expected.

\begin{tabular}{|c|c|c|c|c|c|c|c|c|c|}
\hline$Z$ & Atom & Ground state & $I(\mathrm{eV})$ & $\alpha_{d}$ (a.u.) $)^{\mathrm{a}}$ & $\varepsilon_{b}(\mathrm{eV})^{\mathrm{b}}$ & Excited state(s) & $\varepsilon_{b}(\mathrm{eV})^{\mathrm{c}}$ & $\omega_{\nu}(\mathrm{eV})^{\mathrm{d}}$ & Transition type ${ }^{\mathrm{e}}$ \\
\hline 26 & $\mathrm{Fe}$ & $3 d^{6} 4 s^{25} D_{4}$ & 7.902 & 56.7 & 0.28 & $3 d^{7} 4 s^{5} F_{J}$ & 0.09 & $0.859-0.990$ & $E 2$ \\
\hline 27 & Co & $3 d^{7} 4 s^{24} F_{9 / 2}$ & 7.881 & 50.7 & 0.26 & $3 d^{8} 4 s^{4} F_{J}$ & 0.08 & $0.432-0.582$ & $E 2$ \\
\hline \multirow[t]{3}{*}{28} & $\mathrm{Ni}$ & $3 d^{8} 4 s^{23} F_{4}$ & 7.640 & 45.9 & 0.24 & $3 d^{8} 4 s^{23} F_{2}$ & 0.24 & 0.275 & $E 2$ \\
\hline & & & & & & $3 d^{9} 4 s^{3} D_{1}^{2}$ & 0.07 & 0.212 & $E 2$ \\
\hline & & & & & & $3 d^{9} 4 s^{1} D_{2}$ & 0.07 & 0.423 & $E 2$ \\
\hline 43 & $\mathrm{Tc}$ & $4 d^{5} 5 s^{2}{ }^{6} S_{5 / 2}$ & 7.280 & 77.0 & 0.46 & $4 d^{6} 5 s^{6} D_{J}$ & 0.23 & $0.319-0.518$ & $E 2$ \\
\hline 44 & $\mathrm{Ru}$ & $4 d^{7} 5 s^{5} F_{5}$ & 7.361 & 64.9 & 0.21 & $4 d^{7} 5 s^{5} F_{J}$ & 0.21 & $0.259-0.385$ & $E 2, M 3, E 4$ \\
\hline \multirow[t]{3}{*}{45} & $\mathrm{Rh}$ & $4 d^{8} 5 s^{4} F_{9 / 2}$ & 7.459 & 58.1 & 0.20 & $4 d^{8} 5 s^{4} F_{J}$ & 0.20 & $0.322,0.431$ & $E 2, M 3$ \\
\hline & & & & & & $4 d^{92} D_{J}$ & 0.10 & $0.410,0.701$ & $E 2, M 3$ \\
\hline & & & & & & $4 d^{8} 5 s^{2} F_{7 / 2}$ & 0.20 & 0.706 & $E 2$ \\
\hline 50 & $\mathrm{Sn}$ & $5 s^{2} 5 p^{23} P_{0}$ & 7.344 & 52.0 & 0.02 & $5 s^{2} 5 p^{23} P_{J}$ & 0.02 & $0.210,0.425$ & 0 \\
\hline 051 & $\mathrm{Sb}$ & $5 s^{2} 5 p^{34} S_{3 / 2}$ & 8.608 & 44.6 & 0.05 & $5 s^{2} 5 p^{32} D_{J}$ & 0.05 & $1.055,1.222$ & $E 2$ \\
\hline 73 & $\mathrm{Ta}$ & $5 d^{3} 6 s^{24} F_{3 / 2}$ & 7.550 & 88.5 & 0.45 & $5 d^{3} 6 s^{24} F_{J}$ & 0.45 & $0.249,0.491$ & $E 2$ \\
\hline \multirow[t]{3}{*}{74} & $W$ & $5 d^{4} 6 s^{25} D_{0}$ & 7.864 & 75.0 & 0.46 & $5 d^{4} 6 s^{2}{ }^{5} D_{J}$ & 0.46 & $0.209-0.771$ & $M 1, E 2, M 3, E 4$ \\
\hline & & & & & & $5 d^{5} 6 s^{7} S_{3}$ & 0.30 & 0.366 & M3 \\
\hline & & & & & & $5 d^{4} 6 s^{22} P_{0}$ & 0.46 & 1.181 & $E 0$ \\
\hline \multirow[t]{4}{*}{76} & Os & $5 d^{6} 6 s^{25} D_{4}$ & 8.438 & 57.4 & 0.47 & $5 d^{6} 6 s^{25} D_{J}$ & 0.47 & $0.340-0.755$ & $E 2, M 3, E 4$ \\
\hline & & & & & & $5 d^{7} 6 s^{5} F_{J}$ & 0.29 & $0.638-1.614$ & $E 2, M 3$ \\
\hline & & & & & & $5 d^{6} 6 s^{23} P_{2}$ & 0.47 & 1.260 & $E 2$ \\
\hline & & & & & & $5 d^{7} 6 s^{3} F_{J}$ & 0.29 & $1.368,1.747$ & $E 2$ \\
\hline \multirow[t]{2}{*}{77} & $\mathrm{Ir}$ & $5 d^{7} 6 s^{24} F_{9 / 2}$ & 8.967 & 51.3 & 0.46 & $5 d^{7} 6 s^{22 S+1} L_{J}$ & 0.46 & $0.506-2.204$ & $E 2, M 3, E 4$ \\
\hline & & & & & & $5 d^{8} 6 s^{2 S+1} L_{J}$ & 0.28 & $0.351-2.068$ & $E 2, M 3$ \\
\hline \multirow[t]{3}{*}{78} & $\mathrm{Pt}$ & $5 d^{9} 6 s^{3} D_{3}$ & 8.960 & 43.9 & 0.27 & $5 d^{9} 6 s^{3} D_{J}$ & 0.27 & $0.814,1.256$ & $E 2$ \\
\hline & & & & & & $5 d^{8} 6 s^{22 S+1} L_{J}$ & 0.46 & $1.254-2.106$ & $E 2, M 3$ \\
\hline & & & & & & $5 d^{10{ }^{1}} S_{0}$ & 0.23 & 0.761 & M3 \\
\hline
\end{tabular}

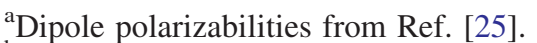

${ }^{\mathrm{b}}$ Binding energies $\varepsilon_{b}=\left|\varepsilon_{0}\right|$ for atoms in ground-state configurations obtained using $\Sigma=\zeta \Sigma^{(2)}$ with $\zeta=2$ (see text).

${ }^{\mathrm{c}}$ Binding energies for atoms in excited-state configurations obtained with $\Sigma=\zeta \Sigma^{(2)}, \zeta=2$.

${ }^{\mathrm{d}}$ Energies of excited states from Ref. [26], such that $0.2 \mathrm{eV}<\omega_{\nu}<E_{\mathrm{Ps}}+0.15 \mathrm{eV}$.

${ }^{\mathrm{e}}$ When several transitions are allowed, the most probable is indicated.

where $\epsilon$ is the mean longitudinal energy of the beam, $k_{\nu}=$ $\sqrt{2 \varepsilon_{\nu}}$, and $\rho_{e p}$ is the electron-positron contact density in the positron bound state, which determines its annihilation width $\Gamma_{\nu}^{a}=\pi r_{0}^{2} c \rho_{e p}$. The function $\Delta$ describes the positron energy distribution around the mean energy $\epsilon, \int \Delta(E) d E=1[28,29]$.

The contact density can be estimated from $\rho_{e p} \approx$ $(F / 2 \pi) \sqrt{2 \varepsilon_{b}}$, where $F=0.66[16,17]$. To evaluate the elastic width, we use a multipole expansion of the positron Coulomb interaction with the atom. Using the fact that for a low-energy positron, large positron-atom separations dominate, one obtains (cf. Ref. [28]),

$$
\Gamma_{\nu}^{e}=\frac{8 k_{\nu}^{2 \lambda}\left|\left\langle\nu J_{\nu}\left\|Q_{\lambda}\right\| 0 J\right\rangle\right|^{2}}{(2 \lambda+1)\left(2 J_{\nu}+1\right)[(2 \lambda+1) ! !]^{2}} f_{\lambda}\left(\varepsilon_{\nu} / \varepsilon_{b}\right),
$$

where $\lambda$ is the order of the multipole (e.g., $\lambda=2$ for a quadrupole excitation), $\left\langle\nu J_{\nu}\left\|Q_{\lambda}\right\| 0 J\right\rangle$ is the reduced matrix element of the $2^{\lambda}$-pole moment between the ground (0) and excited $(\nu)$ atomic states, and $f_{\lambda}(x)=$ $\sqrt{x /(1-x)}\left[{ }_{2} F_{1}\left(\frac{1}{2}, 1 ; \lambda+\frac{3}{2} ;-x\right)\right]^{2}$ is a dimensionless function, such that $f_{\lambda}(x) \sim 1$ for $x \sim 1$.

Estimating $\Gamma_{\nu}^{e}$ from Eq. (4) for a Feshbach resonance at $\varepsilon_{\nu} \sim 1 \mathrm{eV}$, populated through a quadrupole transition, and assuming that the quadrupole amplitude is atomic-sized, one obtains $\Gamma_{\nu}^{e} \sim 1-10 \mathrm{meV}$. Hence, this resonances are sufficiently narrow to produce observable sharp features in the energy dependence of $Z_{\text {eff }}$. Estimating the annihilation width for $\varepsilon_{b}=150 \mathrm{meV}$, we obtain $\Gamma_{\nu}^{a}=4 \times 10^{-7} \mathrm{eV}$, hence, $\Gamma_{\nu}^{e} \gg \Gamma_{\nu}^{a}$. In this case, $\Gamma_{\nu}^{e} / \Gamma_{\nu} \approx 1$, and the contribution of such resonance to $Z_{\text {eff }}$, Eq. (3), is close to maximum possible. For a positron beam with energy spread $\delta \varepsilon \sim 25 \mathrm{meV}$, using $\Delta_{\max } \sim 1 / \delta \varepsilon$, the peak resonant value of the annihilation rate from Eq. (3) is given by $Z_{\text {eff }} \sim$ $\pi F \sqrt{\varepsilon_{b} / \varepsilon_{\nu}} / \delta \varepsilon \sim 10^{3}$. This estimate remains valid even if the elastic width is suppressed by up to 3 orders of magnitude, e.g., for a higher-multipole transition, or a transition mediated by the relativistic (spin-orbit) interaction.

The above analysis indicates that positron-atom resonances could be observed with a trap-based beam used for studying resonances in positron-molecule annihilation [19]. Such a measurement requires vapour pressure of $\sim 0.01$ mtorr [18]. For the atoms in Table I this can be achieved be heating the samples to temperatures ranging from $650{ }^{\circ} \mathrm{C}$ for $\mathrm{Sb}$ and $1100^{\circ} \mathrm{C}$ for $\mathrm{Sn}$, to $1500^{\circ} \mathrm{C}$ for $\mathrm{Fe}, \mathrm{Co}$, and $\mathrm{Ni}$, and over $2000^{\circ} \mathrm{C}$ for other species [30]. Detection of the resonances can thus provide the first experimental evidence of positron binding to neutral 
atoms, and first estimates of the binding energies. While a Feshbach resonance only signifies binding to an excited state, the binding energy in the ground state is expected to be similar if it has the same electronic configuration.

Resonant enhancement can also be observed with thermalized positrons. Depending on the exact position of the resonances, it can lead to a nontrivial dependence of the annihilation rate on the positron temperature, with greater rates measured at higher temperatures. Such behavior would be in sharp contrast with that observed in nonresonant systems, such as the noble gases [31].

One should mention that earlier experimental searches for positron resonances in the vicinity of electronic excitation thresholds (for $\mathrm{H}_{2}, \mathrm{~N}_{2}, \mathrm{CO}$ and $\mathrm{Ar}$ ) yielded negative results [32]. However, these systems are quite different from the open-shell metal atoms considered here. None of them is expected to bind the positron in the ground state, and the electronic excitations lie above the Ps-formation threshold. In addition, the relative role of resonances in the annihilation is much more prominent than in the elastic or total scattering measured in Ref. [32].

The case of transition-metal atoms is also markedly different from that of $\mathrm{Be}$, in which positron binding to the excited $2 s 2 p^{3} P$ state was predicted in configurationinteraction calculations [8]. This excited state lies above the Ps-formation threshold, but a large positron binding energy of $250 \mathrm{meV}$ ensures that the bound state is $40 \mathrm{meV}$ below the $\mathrm{Be}^{+}+$Ps threshold. Such strong binding by the excited state is promoted by its large dipole polarizability. For comparison, the positron binding energy by the ground-state $\mathrm{Be}$ atom is $87 \mathrm{meV}$ [7].

Besides the Feshbach resonances, positron annihilation can be increased by shape resonances. These resonances are supported by the strong polarization attraction and the centrifugal barrier. Thus, calculations predict a sharp $p$-wave resonance in positron scattering from $\mathrm{Mg}$ at $95 \mathrm{meV}$, with $Z_{\text {eff }}=1300$ at the peak, and similar but broader resonances at $\sim 0.45-0.65 \mathrm{eV}$ in $\mathrm{Cu}, \mathrm{Zn}$, and $\mathrm{Cd}$, with $Z_{\text {eff }} \sim 100$ [9,23]. Compared with the Feshbach resonances, the shape resonances do not indicate positron binding. They also have much larger widths, e.g., $\sim 0.1 \mathrm{eV}$ in $\mathrm{Mg}$.

The authors acknowledge useful discussions with C. M. Surko. This work was supported by the Australian Research Council and Gordon Godfrey fund.

*v.dzuba@unsw.edu.au

†v.flambaum@unsw.edu.au

g.gribakin@qub.ac.uk

[1] V. A. Dzuba, V. V. Flambaum, G. F. Gribakin, and W. A. King, Phys. Rev. A 52, 4541 (1995).

[2] G. G. Ryzhikh and J. Mitroy, Phys. Rev. Lett. 79, 4124 (1997).

[3] K. Strasburger and H. Chojnacki, J. Chem. Phys. 108, 3218 (1998).
[4] V.A. Dzuba, V. V. Flambaum, G. F. Gribakin, and C. Harabati, Phys. Rev. A 60, 3641 (1999).

[5] V. A. Dzuba, V. V. Flambaum, and C. Harabati, Phys. Rev. A 62, 042504 (2000).

[6] J. Mitroy, M. W. J. Bromley, and G. G. Ryzhikh, J. Phys. B 35, R81 (2002).

[7] M. W. J. Bromley and J. Mitroy, Phys. Rev. A 73, 032507 (2006).

[8] M. W. J. Bromley and J. Mitroy, Phys. Rev. A 75, 042506 (2007).

[9] J. Mitroy, J. Y. Zhang, M. W. J. Bromley, and S. I. Young, Phys. Rev. A 78, 012715 (2008).

[10] J. Mitroy and G. G. Ryzhikh, J. Phys. B 32, L411 (1999).

[11] J. Mitroy and I. A. Ivanov, J. Phys. B 34, L121 (2001).

[12] J. R. Danielson, J. A. Young, and C. M. Surko, J. Phys. B 42, 235203 (2009).

[13] J. R. Danielson, J. J. Gosselin, and C. M. Surko, Phys. Rev. Lett. 104, 233201 (2010).

[14] S. J. Gilbert, C. Kurz, R. G. Greaves, and C. M. Surko, Appl. Phys. Lett. 70, 1944 (1997); C. Kurz, S. J. Gilbert, R. G. Greaves, and C. Surko, Nucl. Instrum. Methods Phys. Res., Sect. B 143, 188 (1998).

[15] G. F. Gribakin, Phys. Rev. A 61, 022720 (2000).

[16] G. F. Gribakin, in New Directions in Antimatter Chemistry and Physics, edited by C. M. Surko and F. A. Gianturco (Kluwer Academic, Netherlands, 2001), p. 413.

[17] G. F. Gribakin, J. A. Young, and C. M. Surko, Rev. Mod. Phys. 82, 2557 (2010).

[18] S. J. Gilbert, L. D. Barnes, J. P. Sullivan, and C. M. Surko, Phys. Rev. Lett. 88, 043201 (2002).

[19] L. D. Barnes, S. J. Gilbert, and C. M. Surko, Phys. Rev. A 67, 032706 (2003).

[20] M. Ya. Amusia, N. A. Cherepkov, L. V. Chernysheva, and S. G. Shapiro, J. Phys. B 9, L531 (1976).

[21] V. A. Dzuba, V. V. Flambaum, W. A. King, B. N. Miller, and O. P. Sushkov, Phys. Scr. T46, 248 (1993).

[22] J. Mitroy, M. W. J. Bromley, and G. Ryzhikh, J. Phys. B 32, 2203 (1999).

[23] M. W. J. Bromley and J. Mitroy, Phys. Rev. A 81, 052708 (2010).

[24] G.F. Gribakin and J. Ludlow, Phys. Rev. A 70, 032720 (2004).

[25] T. M. Miller, in Handbook of Chemistry and Physics, edited by D. R. Lide (CRC, Boca Raton, 2000).

[26] J. E. Sansonetti, W. C. Martin, and S. L. Young, Handbook of Basic Atomic Spectroscopic Data (NIST, Gaithersburg, MD, 2005).

[27] L. D. Landau and E. M. Lifshitz, Quantum Mechanics (Pergamon, Oxford, 1977), 3rd ed.

[28] G. F. Gribakin and C.M. R. Lee, Phys. Rev. Lett. 97, 193201 (2006).

[29] J. A. Young, G. F. Gribakin, C. M. R. Lee, and C.M. Surko, Phys. Rev. A 77, 060702 (2008).

[30] W.E. Forsythe, Smithsonian Physical Tables (Knovel, 1954, 2003), 9th Rev. ed.

[31] C. Kurz, R. G. Greaves, and C. M. Surko, Phys. Rev. Lett. 77, 2929 (1996).

[32] J.P. Sullivan, S. J. Gilbert, S. J. Buckman, and C.M. Surko, J. Phys. B 34, L467 (2001).

[33] P. Schwerdtfeger and G. A. Bowmaker, J. Chem. Phys. 100, 4487 (1994). 